\title{
Long Noncoding RNA Expression Profiles of Periodontal Ligament Stem Cells from the Periodontitis Microenvironment in Response to Static Mechanical Strain
}

\author{
Jia Liu, ${ }^{1}$ Yan Zhao, ${ }^{1}$ Qiannan Niu, ${ }^{1}$ Ni Qiu, ${ }^{2}$ Shuangyun Liu, ${ }^{3}$ Chunrong Li, $^{1}$ Cuixia Li, ${ }^{1}$ \\ Pei Miao, ${ }^{1}$ Libo Yan, ${ }^{1}$ Qiang Li ${ }^{\circ},{ }^{4}$ and Zuolin Jin $\mathbb{B}$ \\ ${ }^{1}$ State Key Laboratory of Military Stomatology and National Clinical Research Center for Oral Diseases and Shaanxi Clinical \\ Research Center for Oral Diseases, Department of Orthodontics, School of Stomatology, The Fourth Military Medical University, \\ Xi'an, Shaanxi 710032, China \\ ${ }^{2}$ Department of Stomatology, 920 Hospital of PLA, Kunming, Yunnan 650032, China \\ ${ }^{3}$ Department of Stomatology, 260 Hospital of PLA, Shijiazhuang, Hebei 050041, China \\ ${ }^{4}$ State Key Laboratory of Military Stomatology \& National Clinical Research Center for Oral Diseases \& Shaanxi International Joint \\ Research Center for Oral Diseases, Department of General Dentistry \& Emergency, China
}

Correspondence should be addressed to Qiang Li; lqaq726@163.com and Zuolin Jin; zuolinj@163.com

Received 4 October 2020; Revised 1 March 2021; Accepted 8 March 2021; Published 12 April 2021

Academic Editor: Yohei Hayashi

Copyright ( 2021 Jia Liu et al. This is an open access article distributed under the Creative Commons Attribution License, which permits unrestricted use, distribution, and reproduction in any medium, provided the original work is properly cited.

\begin{abstract}
During the period of orthodontic tooth movement, periodontal ligament stem cells (PDLSCs) play an important role in transducing mechanical stimulation and tissue remodeling. However, our previous studies verified that the periodontitis microenvironment causes damage to the biological functions of PDLSCs and abnormal mechanical sensitivity. Long noncoding RNAs (lncRNAs) participate in the inflammatory pathogenesis and development of many diseases. Whether lncRNAs are abnormally expressed in PDLSCs obtained from periodontal tissues of periodontitis patients (PPDLSCs) and whether putative lncRNAs participate in the mechanotransductive process in PDLSCs remain poorly understood. First, we subjected PDLSCs obtained from healthy periodontal tissues (HPDLSCs) and PPDLSCs to static mechanical strain (SMS) with $12 \%$ elongation at $0.1 \mathrm{~Hz}$ frequency using an FX-4000T system and screened overall lncRNA profiles in both cell types by microarray. Among lncRNAs with a fold change (FC) > 20.0, 27 lncRNAs were upregulated in strained HPDLSCs, and 16 lncRNAs (9 upregulated and 7 downregulated) were detected in strained PPDLSCs. For mRNAs with FC $>20.0$, we detected 25 upregulated mRNAs and one downregulated mRNA in strained HPDLSCs and 7 upregulated and 5 downregulated mRNAs in strained PPDLSCs. Further enrichment analysis showed that, unlike HPDLSCs with annotations principally involving transduction-associated signaling pathways, dysregulated mRNAs in PPDLSCs are mainly responsible for pathological conditions. Moreover, coexpressed lncRNA-mRNA networks confirmed the pathological state and exacerbated inflammatory conditions in strained PPDLSCs. Taken together, when compared with strained HPDLSCs, various lncRNAs and mRNAs were dysregulated in PPDLSCs under mechanical forces, implicating the response of lncRNAs in PPDLSCs to mechanical stress. Moreover, we provide potential lncRNA targets, which may contribute to future intervention strategies for orthodontic treatment in periodontitis patients.
\end{abstract}

\section{Introduction}

Periodontitis is a chronic inflammatory disease that causes irreversible periodontal attachment damage [1]. During the pathological process, osteoblasts are distinctly suppressed, whereas osteoclastogenesis becomes hyperactive [2]. Because of the typically high morbidity rate and obvious clinical manifestation of tooth extrusion, space, and labial drifting, many adult patients with periodontitis seek orthodontic treatment to achieve both esthetic restoration and functional 
restoration [3]. Periodontal ligament stem cells (PDLSCs) are considered an attractive source of mesenchymal stem cells (MSCs) in the periodontium and are capable of regenerating cementum/PDL-like structures [4]. However, our previous studies revealed that PDLSCs obtained from periodontal tissues of periodontitis patients (PPDLSCs) are characterized by impaired function that leads to aberrant proliferative and osteogenic properties $[5,6]$.

Mechanical stimuli are another critical factor affecting tissue homeostasis and function. During orthodontic tooth movement, alveolar bone remodeling is triggered via initiation of a series of signaling cascades in the periodontal ligament (PDL) and surrounding tissues [7]. Nonetheless, inappropriate loading can cause homeostasis disruption between osteogenesis and bone resorption [8]. Our previous studies have also demonstrated that the reactions of PPDLSCs and HPDLSCs to static mechanical strain (SMS) differ due to the effect of the inflammatory microenvironment. Specifically, PPDLSCs display a sensitive pattern of both decreased proliferation and osteogenesis and an active inflammatory response to SMS at $12 \%$ elongation, while PDLSCs obtained from healthy periodontal tissues (HPDLSCs) exhibit a notable promotion of multidirectional capacities [6].

Long noncoding RNAs (lncRNAs) are an important type of molecule longer than 200 nucleotides that are transcribed by RNA polymerase II and contain a $5^{\prime}$ cap and $3^{\prime}$ adenylation [9]. Studies have identified that altered lncRNA levels are of functional importance in the pathogenesis and development of various diseases, including skeletal and dental diseases $[10,11]$. In addition, lncRNA remodeling has been demonstrated to affect the progression of periodontitis [12]. For example, our group previously reported that the expression of lncRNA-POIR was reduced in PPDLSCs and that this was accompanied by decreased osteogenic capacity; moreover, overexpression of lncRNA-POIR promoted bone formation by competing with miR-182 [13].

Although many lncRNAs have been identified to be associated with inflammation-induced functional changes, the regulatory effects of lncRNAs on PPDLSCs in response to mechanical forces and the underlying mechanisms remain unclear $[12,14]$. Therefore, this study was aimed at determining the SMS-induced lncRNA profiles of HPDLSCs and PPDLSCs and at exploring potential lncRNAs involved in the process of mechanotransduction in an inflammatory microenvironment.

\section{Materials and Methods}

2.1. Cell Culture. Primary PPDLSCs were obtained from premolar and/or third molar extractions of 8 donors (38.9 \pm 7.9 years old) for therapeutic reasons who were diagnosed with chronic periodontitis. Primary HPDLSCs were isolated from 10 orthodontic patients $(37.9 \pm 7.2$ years old) who underwent routine premolar and/or third molar extractions. All samples were collected at the Department of Orthodontics, School of Stomatology, the Fourth Military Medical University. Periodontitis patients were collected according to the following criteria made by the same periodontal specialist: bleeding on probe; periodontal pocket $<6 \mathrm{~mm}$ with $3-4 \mathrm{~mm}$ attachment loss; and/or alveolar bone absorption up to 1/3$1 / 2$ root length horizontally on $\mathrm{X}$-ray images. None of these subjects were selected with any clinical evidence of systemic disease or an acute infection in the past 6 months, and no one had a smoking history, drug utilization, or ever received maxillofacial radiotherapy and chemotherapy [6, 15]. All subjects provided written informed consent in accordance with the Declaration of Helsinki, and the study reached a consensus with the Ethics Committee of the Fourth Military Medical University (Approval Number: 2017(026)). The primary cells were cultured in $\alpha$-MEM (Gibco BRL, Gaithersburg, MD, USA) with 10\% fetal bovine serum (FBS) (Invitrogen, Carlsbad, CA, USA) in a humidified environment at $37^{\circ} \mathrm{C}$ with $5 \% \mathrm{CO}_{2}$ [14]. Cell colonies were established by the limiting dilution technique [16].

2.2. SMS Loading. All cells were seeded into collagen I-coated 6-well BioFlex plates (Flexcell International, Burlington, NC, USA), and cells were serum starved for $24 \mathrm{~h}$ after achieving 95\% confluence. Experimental cells were subjected to SMS for $12 \mathrm{~h}$ utilizing a Flexcell Tension Plus system (FX-4000T, Flexcell International) with $12 \%$ elongation at $0.1 \mathrm{~Hz}$ [6]. Static groups were cultured under the same conditions without SMS exposure.

2.3. RNA Extraction. Total RNA was extracted using TRIzol reagent (Invitrogen, Carlsbad, CA, USA) and quantified using a NanoDrop ND-1000 (Thermo Fisher Scientific, Boston, MA, USA). RNA integrity was evaluated by standard denaturing agarose gel electrophoresis.

2.4. Microarray Analysis. Sample labeling and array hybridization were conducted using the Agilent One-Color Microarray-Based Gene Expression Analysis protocol (Agilent Technologies, Santa Clara, CA, USA) [13]. In brief, mRNA was purified from total RNA after removing rRNA (mRNA-ONLY Eukaryotic mRNA Isolation Kit; Epicenter, San Diego, CA, USA). Each sample was amplified and transcribed into fluorescent cRNA along the entire length of the transcripts without $3^{\prime}$ bias via a random priming method (Arraystar Flash RNA Labeling Kit; Arraystar, Rockville, $\mathrm{MD}$, USA). The labeled cRNAs were purified using an RNeasy Mini Kit (Qiagen, Hilden, Germany). The concentration and specific activity of the labeled cRNAs (pmol Cy3/ $\mu$ g cRNA) were assessed using a NanoDrop ND-1000. One microgram of each labeled cRNA was fragmented by adding $5 \mu \mathrm{l}$ of $10 \times$ blocking agent and $1 \mu \mathrm{l}$ of $25 \times$ fragmentation buffer; after heating at $60^{\circ} \mathrm{C}$ for $30 \mathrm{~min}, 25 \mu \mathrm{l}$ of $2 \times \mathrm{GE}$ hybridization buffer was added to dilute the labeled cRNA. Approximately $50 \mu \mathrm{l}$ of hybridization solution was dispensed into the gasket slide and assembled onto the lncRNA expression microarray slide. The slides were incubated for 17 at $65^{\circ} \mathrm{C}$ in an Agilent hybridization oven (Agilent, Santa Clara, CA, USA). The hybridized arrays were washed, fixed, and scanned using an Agilent DNA Microarray Scanner (part number G2505C). Arraystar Human LncRNA Microarray V3.0 (Arraystar, Kangchen, Shanghai, China) was designed for the global profiling of human lncRNAs and mRNAs. Approximately 30,586 lncRNAs and 26,109 coding transcripts 
can be detected by collecting data sources from GENCODE, UCSC, Ensembl, RefSeq, and other related sources.

2.5. Real-Time qPCR Confirmation. Total RNA was assembled and reverse-converted to cDNA using a SuperScript First-Strand Synthesis Kit (Invitrogen). An Applied Biosystems ViiA 7 Real-Time PCR System was used for qPCR. The reaction system included incubation for $10 \mathrm{~min}$ at $95^{\circ} \mathrm{C}$, followed by 40 cycles at $95^{\circ} \mathrm{C}$ for $10 \mathrm{~s}$ and $60^{\circ} \mathrm{C}$ for $1 \mathrm{~min}$. Relative expression levels of transcripts were calculated by using the $2^{-\Delta \Delta \mathrm{CT}}$ method and normalized to GAPDH [17]. All experiments were carried out in triplicate. The specific primers (Genscript, China) used are shown in Table 1.

2.6. Bioinformatic Analysis of Differentially Expressed (DE) $m R N A s$. Gene Ontology (GO) analysis was employed to map DEmRNAs to GO terms annotated by molecular function, biological process, and cellular components (http:// www.geneontology.org). Significant pathways of the DE genes were determined using Kyoto Encyclopedia of Genes and Genomes (KEGG) (http://www.genome.jp/kegg/), as previously described $[18,19]$.

2.7. Coexpression Network Construction (CNC). CNC was conducted based on the top 10 DElncRNAs between strained HPDLSCs and PPDLSCs with coexpressed DEmRNAs [20]. Pearson's correlation coefficients (PPCs) no less than 0.99 were used to identify coding genes. CNCs were accomplished using Cytoscape software version 3.0.1 (The Cytoscape Consortium, San Diego, CA, USA).

2.8. Lentivirus Transfection. The design and construction of lentiviruses were performed by GeneChem (GeneChem, Shanghai, China). The lentivirus Ubi-MCS-SV40-EGFPIRES-puromycin was used for lncRNA-XIST overexpression, and hU6-MCS-CBh-gcGFP-IRES-puromycin was used for lncRNA-XIST interference. The sequences of primers for amplifying lncRNA-XIST were F: $5^{\prime}$-ACAAGCAGTGC AGAGAGCT- ${ }^{\prime}$ and R: $5^{\prime}$-AGAGTGCCAGGCATGT TGA- ${ }^{\prime}$. The sequences of IncRNA-XIST interference targets were as follows: shlncRNA-XIST (79428-1): 5'-GCCATC ATTAGCCACTGCACT- ${ }^{\prime}$; shlncRNA-XIST (79428-2): $5^{\prime}$ -GGTCAGGAGGTTCTGTCAAGA-3'; and shlncRNAXIST (79428-3): 5'-GGTCCCAGATAGGAAGATAAA-3' . HPDLSCs and PPDLSCs were cultured in six-well plates. When cells reached approximately $30 \%$ confluence, they were transfected with lentiviruses at a multiplicity of transfection (MOI) of 9 for $24 \mathrm{~h}$ and then cultured with common medium ( $\alpha$-MEM with $10 \%$ FBS).

2.9. Osteogenic Differentiation Assays. For osteogenesis assays, HPDLSCs and PPDLSCs were exposed to SMS with $12 \%$ elongation at $0.1 \mathrm{~Hz}$ for $12 \mathrm{~h}$ after reaching $80 \%$ confluence. Then, HPDLSCs and PPDLSCs were cultured in osteogenic medium for 21 days. Mineralized nodules were stained with alizarin red S ( $\mathrm{pH} 4.2)$ (Kermel, Tianjin, China) for $15 \mathrm{~min}$ at room temperature at day 21 , and calcium levels were measured quantitatively using a calcium colorimetric assay kit (BioVision, San Francisco, CA, USA).
TABLE 1: Primers designed for real-time qPCR validation of candidate lncRNAs.

\begin{tabular}{lc}
\hline Gene symbol & \multicolumn{1}{c}{ Sense primer } \\
\hline \multirow{2}{*}{ GAPDH (HUMAN) } & F: 5'GGGAAACTGTGGCGTGAT3' \\
R: 5' GAGTGGGTGTCGCTGTTGA3' \\
TCONS_00008604 & F: 5' GTTGGGCAGTAAGCCTCACA3' \\
& R: 5' TGGGGTAGGTAATGGAAAAAG3' \\
ENST00000428781 & F: 5' AGGGGGTAAAAGAAAATGGTG3' \\
& R: 5'CAGGCTCGCATTCAGACAT3' \\
uc004arq.1 & F: 5' ACCCCTACAGACCATAACAAAG3' \\
& R: 5' AGCCGACTACAGCCACCACT3' \\
XIST & F: 5'GCTGAATGAATGTGTCTTACCC3' \\
& R: 5'GAGGCAAAGGCACACACGAA3' \\
Runx2 & F: 5'CCCGTGGCCTTCAAGGT-3' \\
& R: 5'CGTTACCCGCCATGACAGTA-3' \\
\hline
\end{tabular}

2.10. Statistical Analysis. All experiments were performed in triplicate, and the data are presented as the means \pm standard deviation (S.D.). Statistical analyses with one-way ANOVA and Student's $t$-test were performed using SPSS 16.0 software (SPSS, San Rafael, CA, USA). Correlated terms were performed with the PCCs, and the significance threshold was fold change (FC) $\geq 2.0$ and PCCs $\geq 0.9$ and/or $P<0.05$.

\section{Results}

3.1. Expression Profiles of DElncRNAs and DEmRNAs with SMS. According to the principles of FC $\geq 2.0$ and PPCs $\geq$ 0.9 , we screened 8,847 and 9,772 DElncRNAs in strained HPDLSCs and PPDLSCs relative to static controls, respectively (Figure 1(a)). In addition, 1,624 DElncRNAs were only expressed in strained HPDLSCs, and 2,549 were only expressed in strained PPDLSCs. DElncRNAs with FC > 20.0 in each group are provided in Tables 2 and 3. Of those, ENST00000411904 was the most upregulated lncRNA in strained HPDLSCs; the most upregulated and downregulated lncRNAs in strained PPDLSCs were lncRNA-XIST and ENST00000517505, respectively. Volcano and scatter plots as well as hierarchical clustering were examined to assess the lncRNA expression differences between HPDLSCs and PPDLSCs exposed to the strain (Figures 1(b)-1(d)).

Thousands of DEmRNAs were identified (Figure 1(e)). In total, 11,937 and 12,410 DEmRNAs were significantly altered in strained HPDLSCs and PPDLSCs, respectively. In particular, 2,170 specific DEmRNAs were detected in strained HPDLSCs and 2,643 in strained PPDLSCs. The most upregulated and downregulated mRNAs in strained HPDLSCs were ASHGA5P006667 and ASHGA5P003418, and the most upregulated and downregulated mRNAs in strained PPDLSCs were ASHGA5P009176 and ASHGA5P052412 (KIF20A) (Tables 4 and 5). The volcano and scatter plots depicted in Figures 1(f) and 1(g) demonstrate the variation in lncRNA expression between strain-induced HPDLSCs and PPDLSCs. 


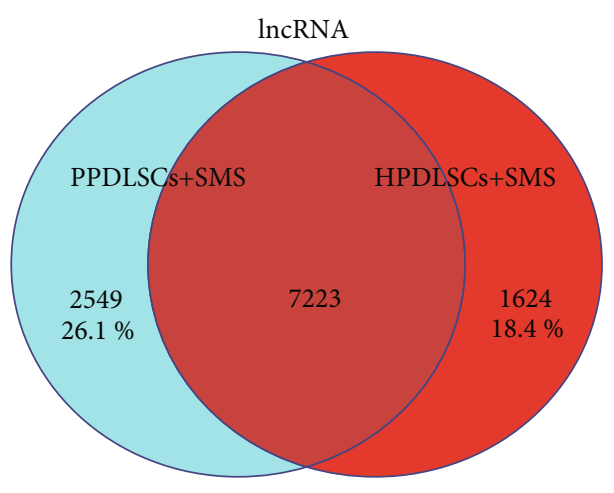

(a)

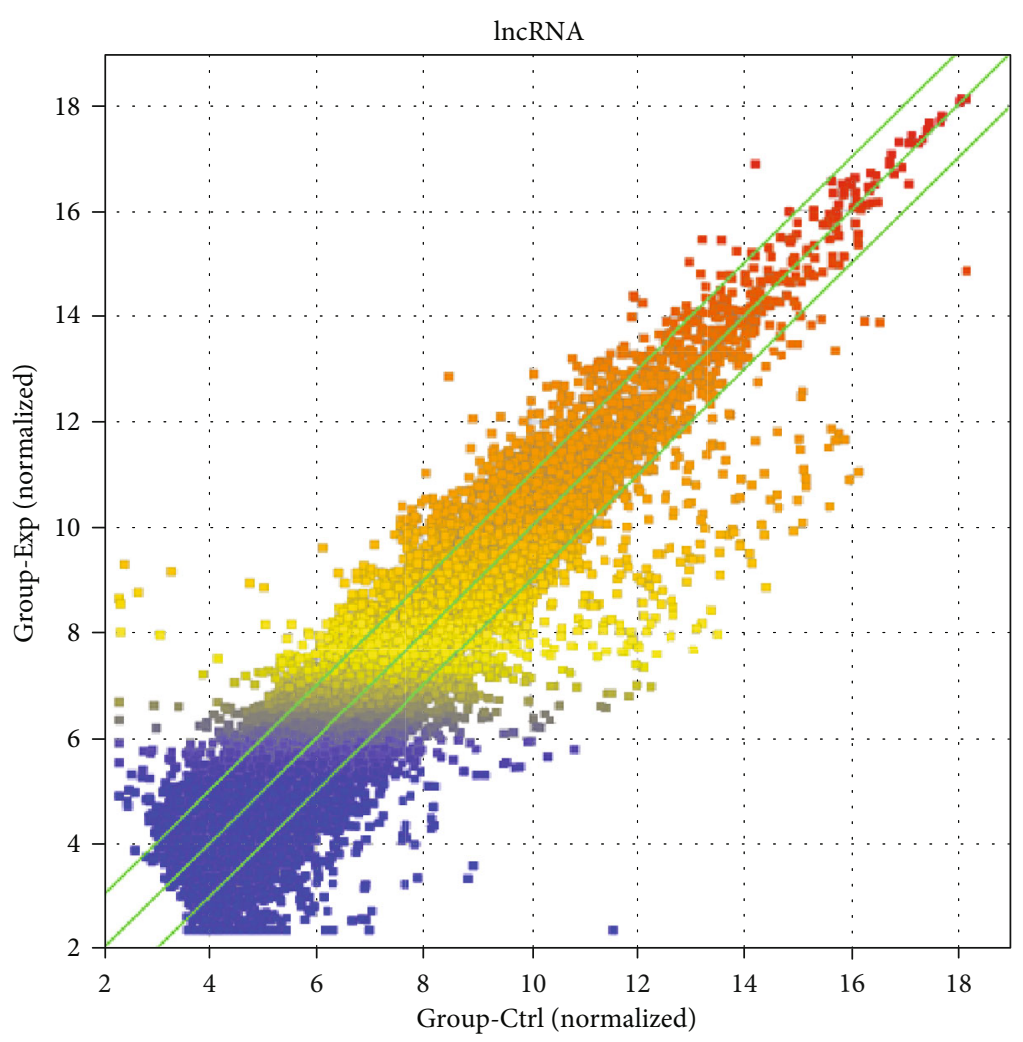

(c)

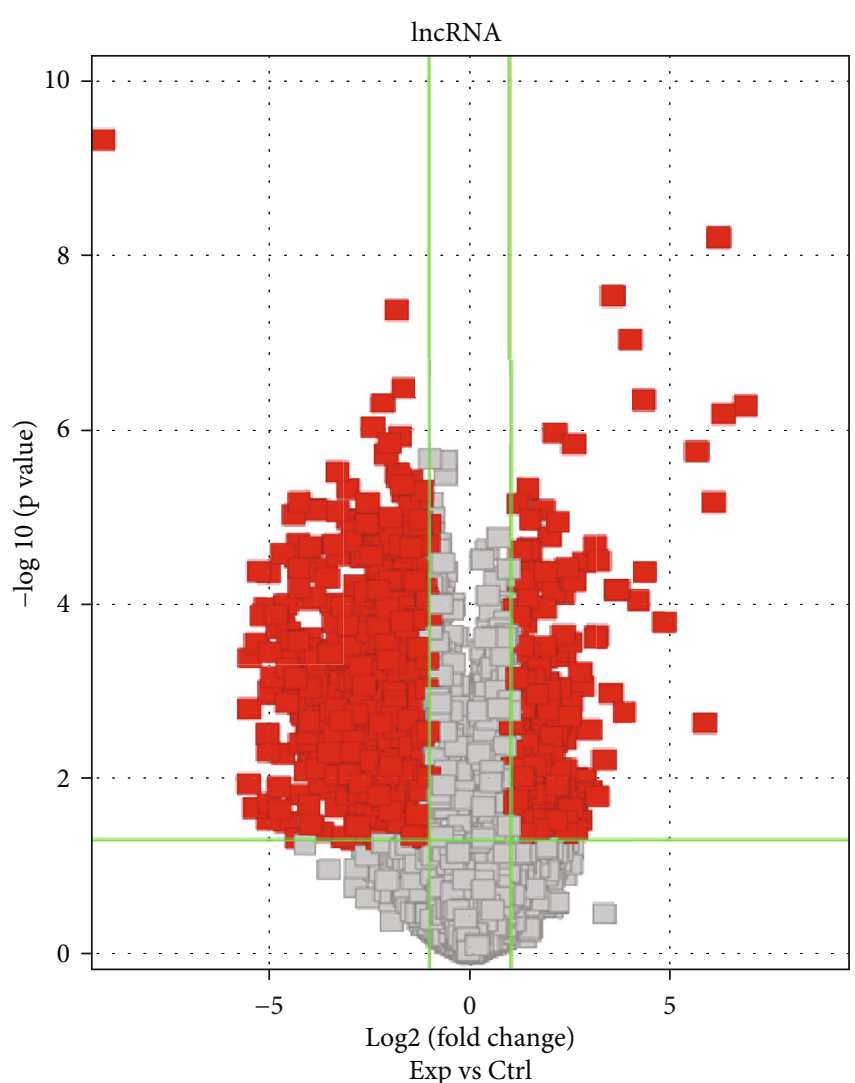

(b)
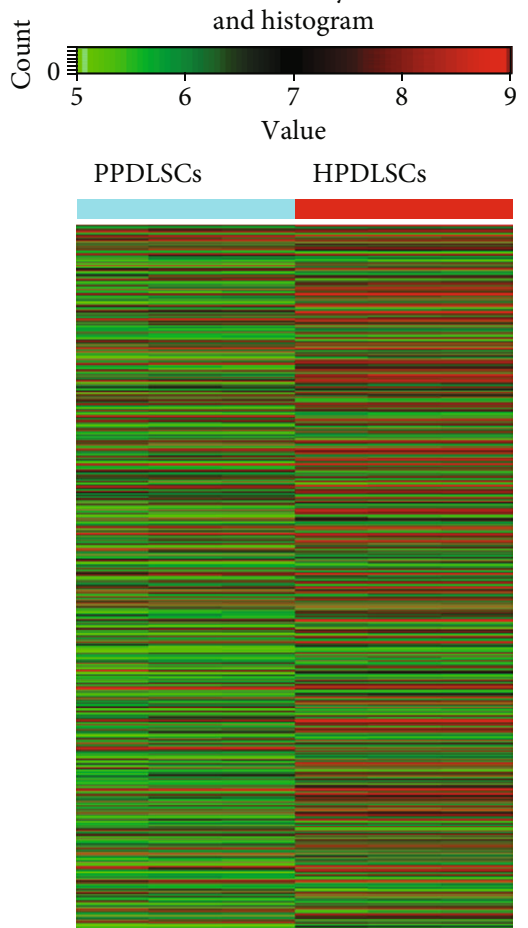

$\begin{array}{llllll}\text { PP1 } & \text { PP2 } & \text { PP3 } & \text { HP1 HP2 } & \text { HP3 }\end{array}$

(d)

Figure 1: Continued. 


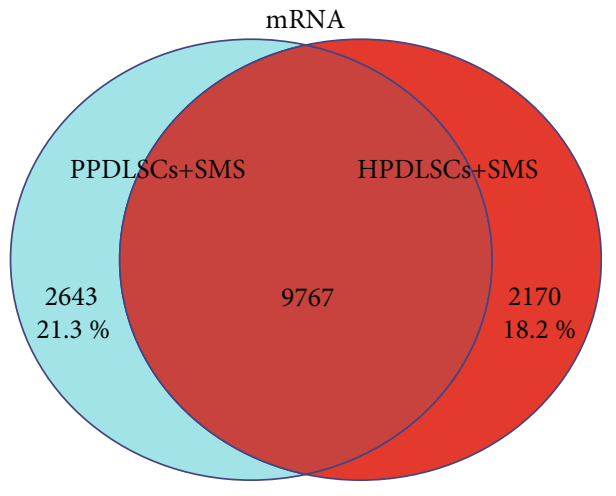

(e)

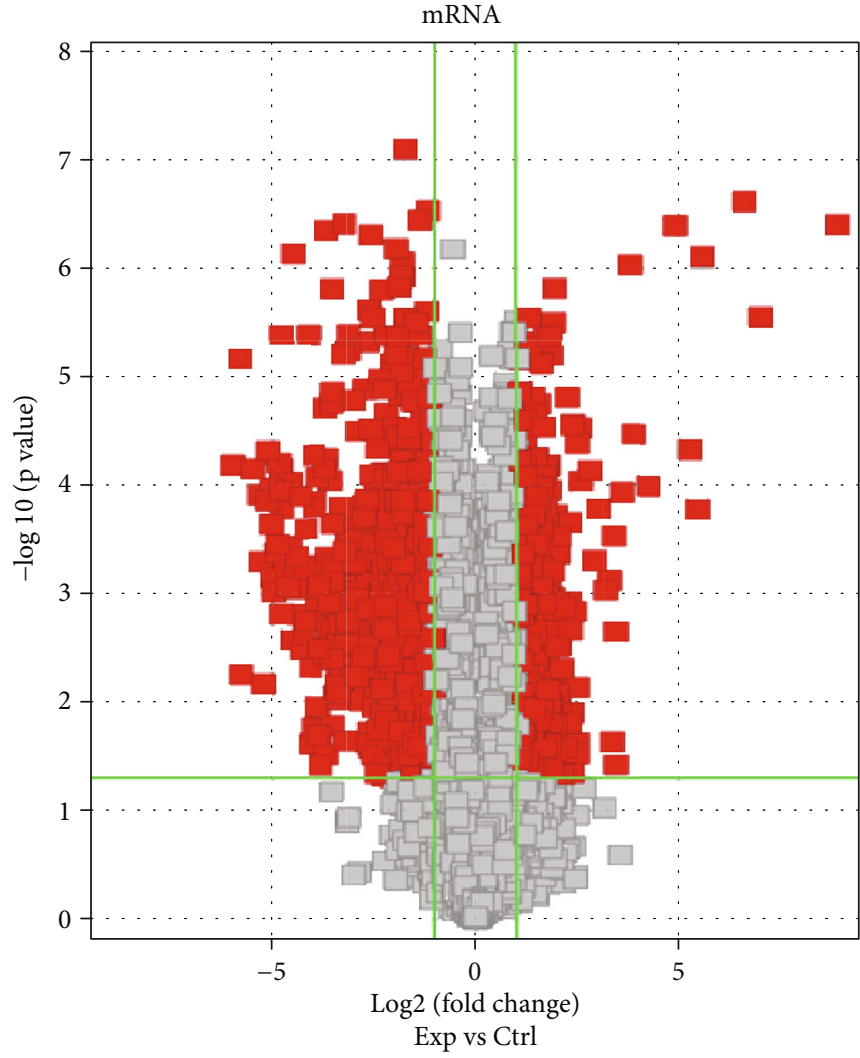

(f)

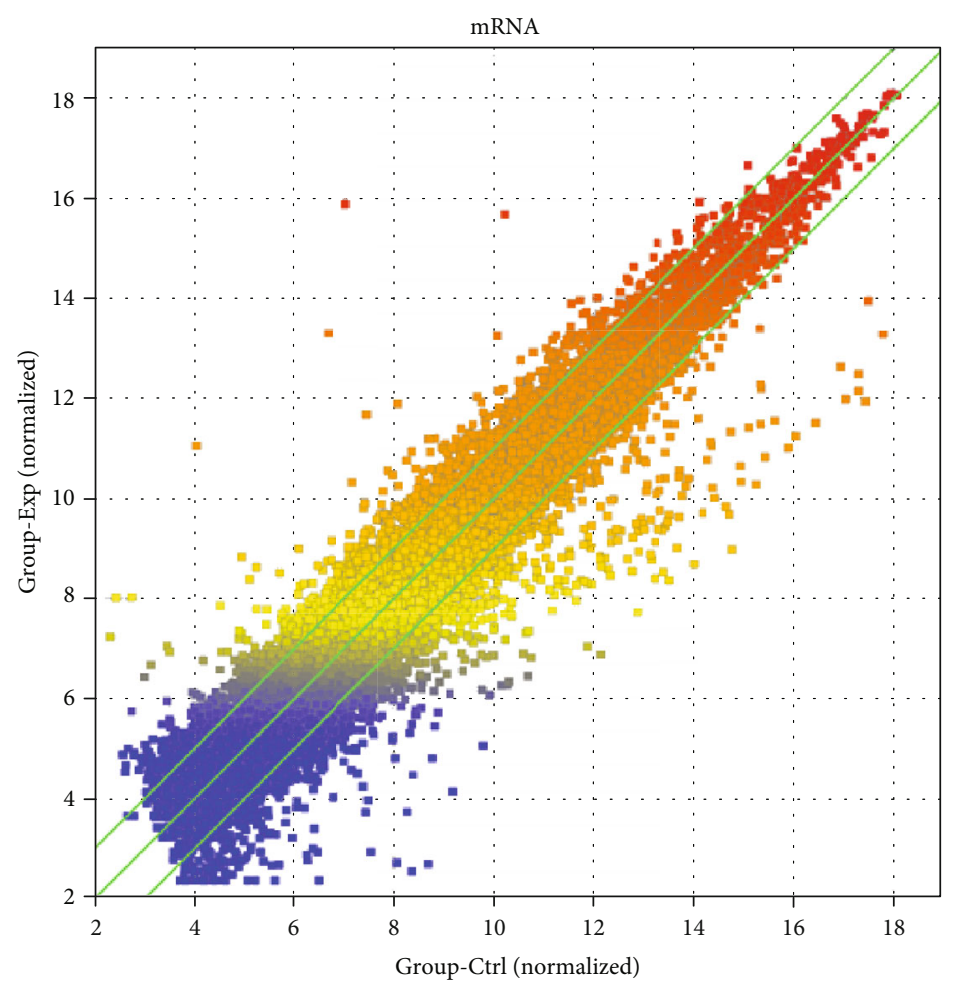

(g)

Figure 1: DElncRNAs and DEmRNAs between HPDLSCs and PPDLSCs after SMS exposure. Venn diagrams of DElncRNAs (a) and DEmRNAs (e). Volcano plot of expression profiles of lncRNAs (b) and mRNAs (f). Scatter plot of expression variations of lncRNAs (c) and mRNAs (g). Dots above the top and below the bottom green lines represent FC > 4.0. (d) Hierarchical clustering of DElncRNAs. Red represents relatively high expression, and green represents relatively low expression. 
TABLE 2: DElncRNAs with FC > 20.0 in strained HPDLSCs compared to static controls.

\begin{tabular}{|c|c|c|c|c|}
\hline Sequence name & Source & Fold change & Regulation & $P$ value $\left(\times 10^{-4}\right)$ \\
\hline NR_038400 & RefSeq & 35.06 & Up & 0.000 \\
\hline ENST00000423442 & GENCODE & 35.06 & Up & 0.024 \\
\hline ENST00000409139 & GENCODE & 33.15 & Up & 0.229 \\
\hline ENST00000432511 & GENCODE & 31.93 & Up & 0.039 \\
\hline ENST00000433431 & Pseudogene & 31.57 & Up & 0.169 \\
\hline ENST00000453278 & GENCODE & 30.70 & Up & 0.358 \\
\hline uc003 mjk.3 & UCSC_knowngene & 29.42 & Up & 24.755 \\
\hline ENST00000447956 & GENCODE & 28.82 & Up & 0.047 \\
\hline ENST00000505532 & GENCODE & 28.06 & Up & 0.235 \\
\hline TCONS_00024645 & LincRNAs identified by Cabili et al. & 27.71 & Up & 0.451 \\
\hline TCONS_00010599 & LincRNAs identified by Cabili et al. & 24.92 & Up & 0.659 \\
\hline DB299803 & LincRNAs identified by Khalil et al. & 23.18 & Up & 0.115 \\
\hline TCONS_00008978 & LincRNAs identified by Cabili et al. & 21.99 & Up & 0.784 \\
\hline ENST00000506014 & GENCODE & 20.49 & Up & 1.714 \\
\hline
\end{tabular}

TABLE 3: DElncRNAs with FC > 20.0 in strained PPDLSCs compared to static controls.

\begin{tabular}{|c|c|c|c|c|}
\hline Sequence name & Source & Fold change & Regulation & $P$ value $\left(\times 10^{-4}\right)$ \\
\hline XIST & GENCODE & 53.85 & Down & 1.089 \\
\hline ENST00000517505 & GENCODE & 50.01 & Up & 107.825 \\
\hline ENST00000583761 & GENCODE & 40.94 & Up & 5.450 \\
\hline TCONS_00024405 & LincRNAs identified by Cabili et al. & 37.30 & Down & 0.059 \\
\hline ENST00000523905 & GENCODE & 36.91 & $\mathrm{Up}$ & 20.468 \\
\hline TCONS_00008604 & LincRNAs identified by Cabili et al. & 34.05 & Down & 0.795 \\
\hline AA324424 & LincRNAs identified by Khalil et al. & 33.45 & $\mathrm{Up}$ & 0.048 \\
\hline ENST00000545920 & GENCODE & 33.25 & Down & 3.759 \\
\hline ENST00000486545 & GENCODE & 27.35 & Down & 0.003 \\
\hline TCONS_00019524 & LincRNAs identified by Cabili et al. & 25.44 & Up & 4.413 \\
\hline ENST00000428781 & GENCODE & 24.73 & Down & 0.259 \\
\hline uc004arq.1 & UCSC_knowngene & 22.42 & Down & 0.059 \\
\hline TCONS_00014003 & LincRNAs identified by Cabili et al. & 21.10 & Up & 20.461 \\
\hline
\end{tabular}

3.2. Confirmation of DElncRNAs Using Real-Time qPCR. To validate the microarray results, we randomly selected four lncRNAs (TCONS_00008604, ENST00000428781, uc004arq.1, and XIST) from the top 10 DElncRNAs between strained HPDLSCs and PPDLSCs and evaluated their expression by qPCR assay (Table 6). All lncRNAs were downregulated in strained PPDLSCs compared to HPDLSCs, which was consistent with the microarray analysis results (Figure 2).

3.3. Preliminary Analysis of DEmRNAs with SMS. To further explore the putative functions of lncRNAs, bioinformatic analysis was applied based on GO and KEGG pathway analyses. According to the results, DEmRNAs in strained HPDLSCs were mainly enriched in the regulation of stress response, signal transduction, and response to stimulus (Figures 3(a), 3(c), and 3(e)). In contrast, pathological processes such as cell-type apoptotic processes and neuronal apoptotic processes were enriched in strained PPDLSCs
(Figures 3(b), 3(d), and 3(f)). Enrichment scores revealed prominent assignments for cellular function and metabolism, such as fatty acid degradation and metabolism, in strained HPDLSCs (Figure 3(g)). In strained PPDLSCs, pathological states were largely notable, including Huntington's disease, bladder cancer, and non-small-cell lung cancer (Figure 3(h)).

3.4. Constructions of the CNC Network. By combining the top 10 DElncRNAs with coexpressed DEmRNAs, an integrated coexpression network containing 1,250 lncRNA-mRNA interactions was established (Figure 4(a)). Notably, RP11-597D13.9 (ENST00000505532) was associated with the maximum number of DEmRNAs, up to 160. XIST, the most downregulated lncRNA in strained PPDLSCs, was coexpressed with 47 DEmRNAs (Table 6). In addition, GO annotations and KEGG analyses showed that the DElncRNAs in the key module are related to chondrocyte development, fibroblast apoptotic process regulation, and cell adhesion as well as leukocyte transendothelial migration, which likely participate in tissue 
TABLE 4: DEmRNAs with $\mathrm{FC}>20.0$ in strained HPDLSCs compared to static controls.

\begin{tabular}{|c|c|c|c|c|}
\hline Sequence name & Source & $\begin{array}{c}\text { Fold } \\
\text { change }\end{array}$ & Regulation & $\begin{array}{l}P \text { value } \\
\left(\times 10^{-5}\right)\end{array}$ \\
\hline ASHGA5P006667 & RefSeq & 413.31 & Up & 4.315 \\
\hline ASHGA5P008770 & RefSeq & 147.59 & Up & 0.0003 \\
\hline ASHGA5P021973 & RefSeq & 125.83 & Up & 150.671 \\
\hline ASHGA5P013772 & GENCODE & 70.35 & Up & 1512.671 \\
\hline ASHGA5P003418 & RefSeq & 63.94 & Down & 74.253 \\
\hline ASHGA5P011737 & RefSeq & 42.60 & Up & 494.192 \\
\hline ASHGA5P007165 & GENCODE & 41.99 & Up & 302.842 \\
\hline ASHGA5P037277 & RefSeq & 41.81 & Up & 1.06 .612 \\
\hline ASHGA5P005733 & GENCODE & 38.72 & Up & 46.774 \\
\hline ASHGA5P002830 & GENCODE & 37.77 & Up & 15.020 \\
\hline ASHGA5P002962 & RefSeq & 36.22 & Up & 5.402 \\
\hline ASHGA5P013771 & RefSeq & 33.74 & Up & 46.840 \\
\hline ASHGA5P042689 & RefSeq & 32.07 & Up & 92.410 \\
\hline ASHGA5P002150 & RefSeq & 31.42 & Up & 0.128 \\
\hline ASHGA5P007745 & RefSeq & 30.45 & Up & 6.915 \\
\hline ASHGA5P003294 & GENCODE & 28.31 & Up & 0.254 \\
\hline ASHGA5P017399 & RefSeq & 28.24 & Up & 7.840 \\
\hline ASHGA5P008733 & GENCODE & 25.41 & Up & 64.712 \\
\hline ASHGA5P005403 & RefSeq & 23.76 & Up & 1.361 \\
\hline ASHGA5P054134 & RefSeq & 23.62 & Up & 245.163 \\
\hline ASHGA5P001729 & RefSeq & 23.04 & Up & 14.825 \\
\hline ASHGA5P051262 & RefSeq & 22.99 & $\mathrm{Up}$ & 858.448 \\
\hline ASHGA5P045542 & RefSeq & 22.91 & $\mathrm{Up}$ & 200.313 \\
\hline ASHGA5P050260 & RefSeq & 22.54 & $\mathrm{Up}$ & 0.001 \\
\hline ASHGA5P004313 & RefSeq & 21.00 & $\mathrm{Up}$ & 4.695 \\
\hline ASHGA5P001728 & RefSeq & 20.64 & Up & 84.501 \\
\hline
\end{tabular}

TABLE 5: DEmRNAs with FC > 20.0 in strained PPDLSCs compared to static controls.

\begin{tabular}{lcccc}
\hline Sequence name & Source & $\begin{array}{c}\text { Fold } \\
\text { change }\end{array}$ & Regulation & $\begin{array}{c}P \text { value } \\
\left(\times 10^{-5}\right)\end{array}$ \\
\hline ASHGA5P009176 & RefSeq & 111.34 & $\mathrm{Up}$ & 179.785 \\
ASHGA5P013422 & RefSeq & 59.61 & $\mathrm{Up}$ & 6.424 \\
ASHGA5P010424 & GENCODE & 56.12 & $\mathrm{Up}$ & 0.032 \\
ASHGA5P012978 & RefSeq & 42.19 & $\mathrm{Up}$ & 104.171 \\
ASHGA5P052412 & RefSeq & 42.10 & Down & 0.001 \\
ASHGA5P003780 & RefSeq & 34.88 & $\mathrm{Up}$ & 2427.963 \\
ASHGA5P005903 & RefSeq & 31.08 & Down & 0.919 \\
ASHGA5P017401 & RefSeq & 27.31 & Down & 0.304 \\
ASHGA5P001619 & RefSeq & 25.51 & Up & 784.373 \\
ASHGA5P004428 & RefSeq & 24.18 & Down & 26.041 \\
ASHGA5P004064 & RefSeq & 21.49 & Down & 4.546 \\
ASHGA5P034395 & GENCODE & 20.98 & Up & 55.833 \\
\hline
\end{tabular}

regeneration. Taken together, dysregulated lncRNAs are involved in the pathological modification of gene expression in PPDLSCs under mechanical conditions (Figures 4(b)-4(e)).
TABLE 6: Top 10 DElncRNAs between strained HPDLSCs and PPDLSCs with coexpressed mRNAs.

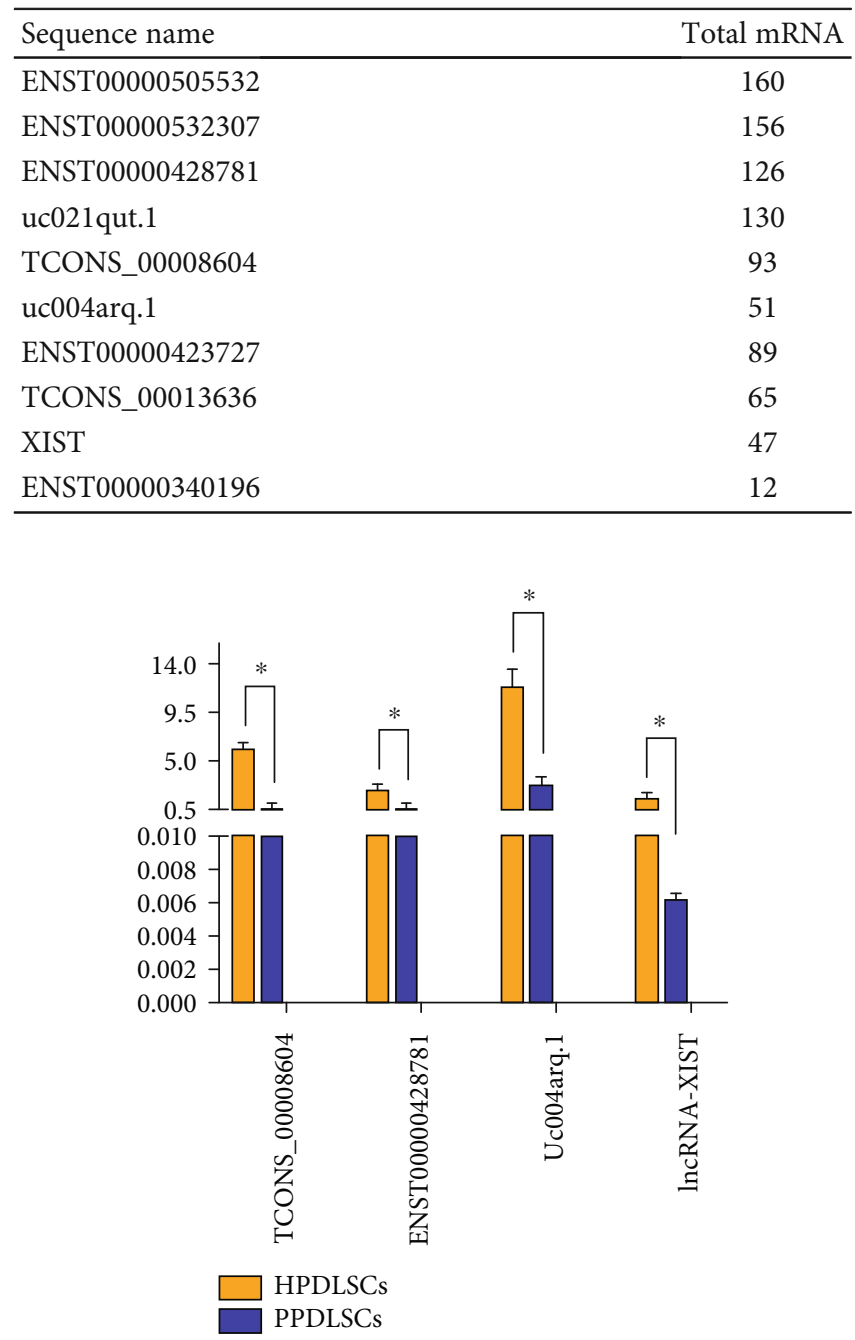

FIgURE 2: Real-time qPCR conformation of DE lncRNAs between strained HPDLSCs and PPDLSCs. All experiments were performed in triplicate, and the data are presented as the mean $\pm \mathrm{S}$ .D. ${ }^{*} P<0.05$.

3.5. Functional Investigation of DElncRNAs during Osteogenic Differentiation. We randomly evaluated the expression of four lncRNAs (TCONS_00008604, ENST00000428781, uc004arq.1, and XIST) from the top 10 DElncRNAs between strained HPDLSCs and PPDLSCs after osteogenic induction for 7 days and observed that the expression level of lncRNA-XIST significantly increased in HPDLSCs at day 7 after osteogenic differentiation. Although osteogenic induction upregulated the level of lncRNA-XIST in PPDLSCs, it was still lower than that in HPDLSCs $(P<0.05$, Figure 5(a)). We also found that lncRNA-XIST was significantly increased in HPDLSCs after $12 \mathrm{~h}$ of SMS elongation $(P<0.05)$; however, strain-induced lncRNA-XIST expression was not obviously increased in PPDLSCs (Figure 5(b)). We also examined the relationship between lncRNA-XIST and the osteogenic gene Runx2 after 12\% SMS loading by lentivirus transfection. We found that Runx2 

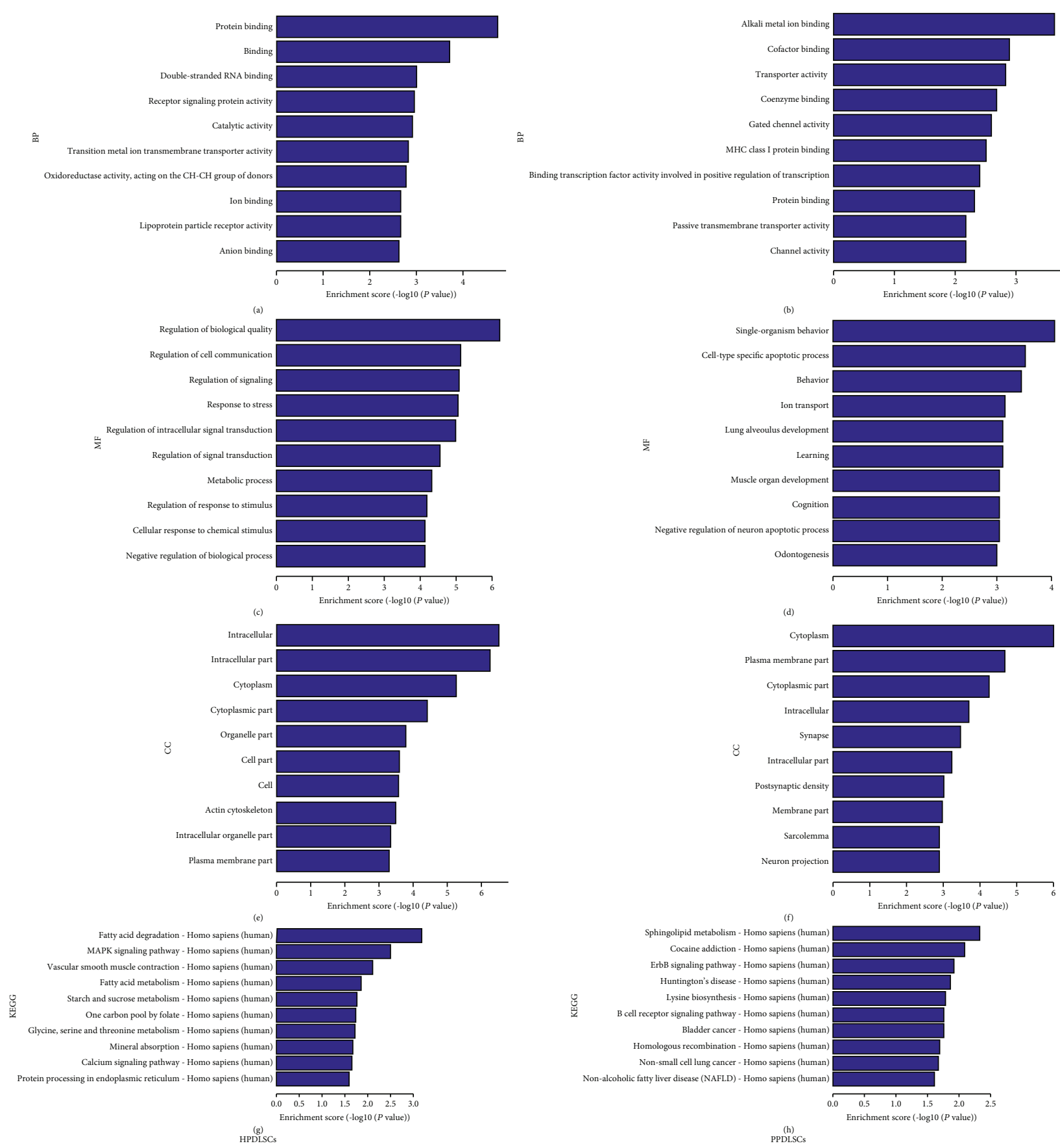

Figure 3: Top 10 functional analyses of DEmRNAs in strained HPDLSCs and PPDLSCs compared to static controls. Biological processes of DEmRNAs in strained HPDLSCs (a) and PPDLSCs (b). Molecular functions of DEmRNAs in strained HPDLSCs (c) and PPDLSCs (d). Cellular components of DEmRNAs in strained HPDLSCs (e) and PPDLSCs (f). KEGG pathways of DEmRNAs in strained HPDLSCs (g) and PPDLSCs (f).

expression in strained HPDLSCs infected with shlncRNAXIST was decreased almost 2 times compared with strained HPDLSCs in the negative control group (NC) $(P<0.05$, Figure 5(c)). In contrast, Runx2 expression in strained PPDLSCs increased after lncRNA-XIST overexpression $(P<0.05$, Figure 5(d)). Similarly, alizarin red staining and calcium quantification also sustained that shlncRNA-XIST inhibited SMS-induced osteogenic differentiation in HPDLSCs and that overexpression of lncRNA-XIST rescued the osteogenic ability of PPDLSCs $(P<0.05$, Figures 5(e) and 5(f)).

\section{Discussion}

Many lncRNAs play critical roles in multiple pathological processes of periodontitis, such as proliferation, differentiation, cell migration, and immune regulation $[21,22]$. 


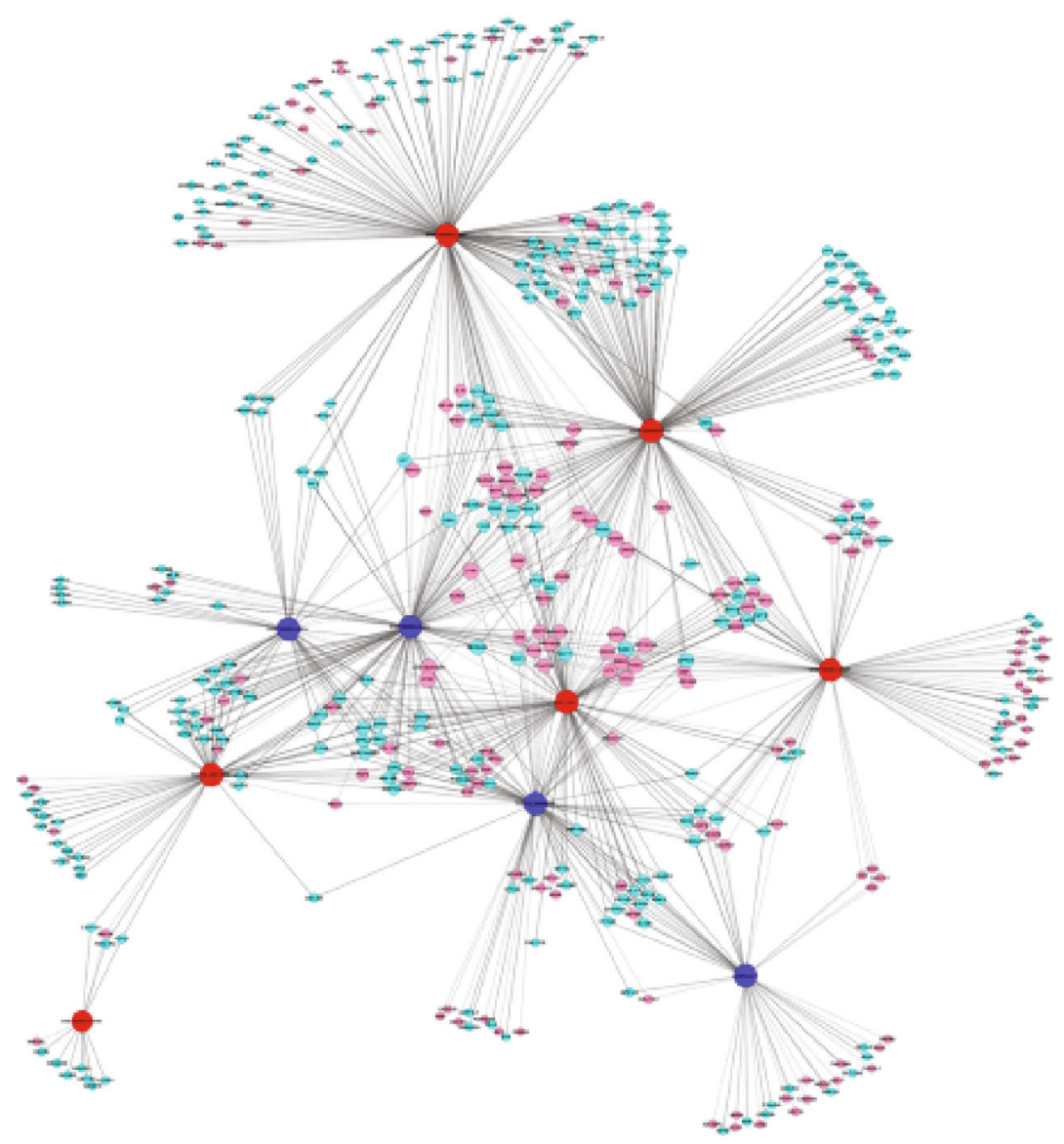

(a)

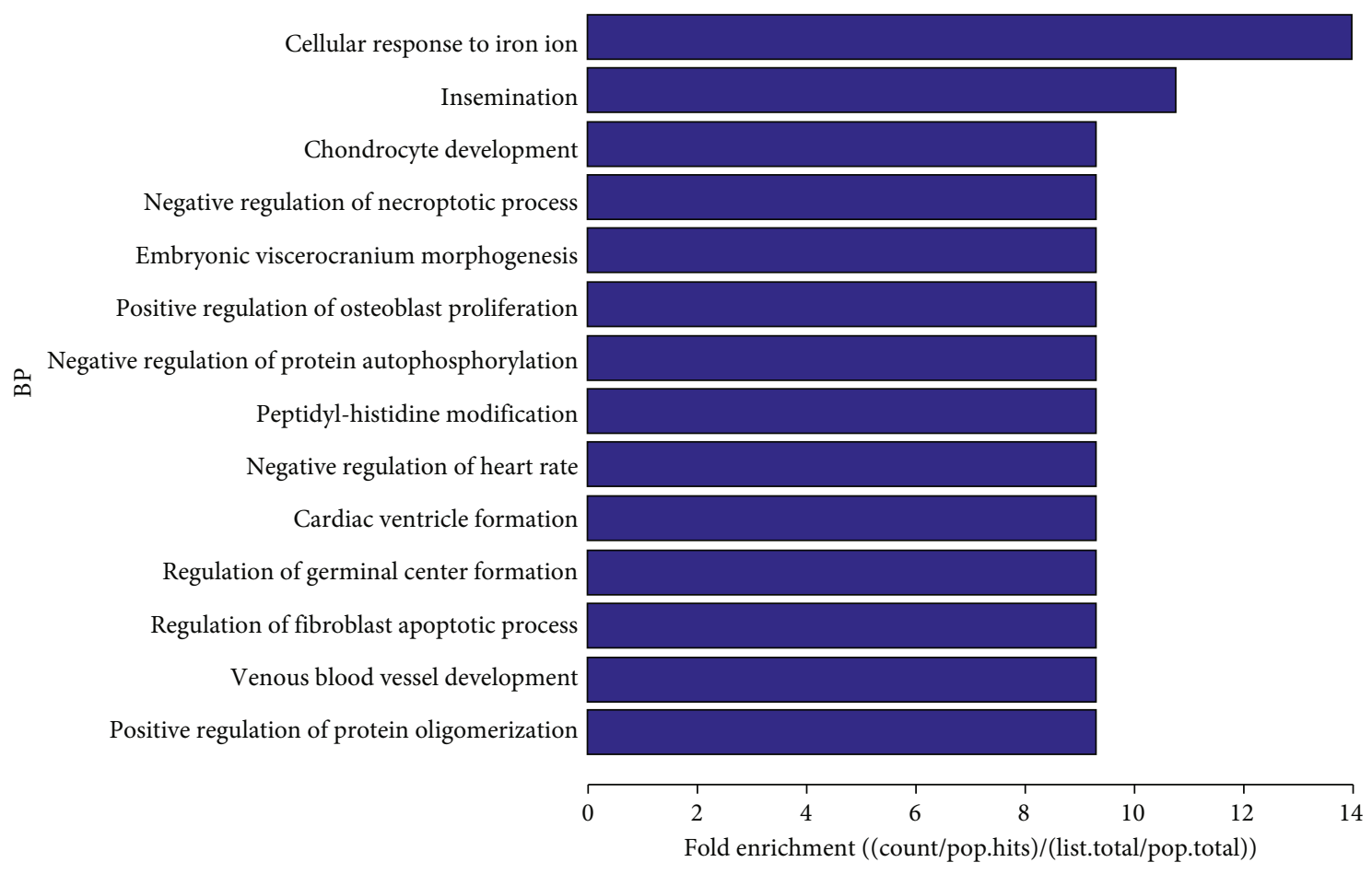

(b)

Figure 4: Continued. 


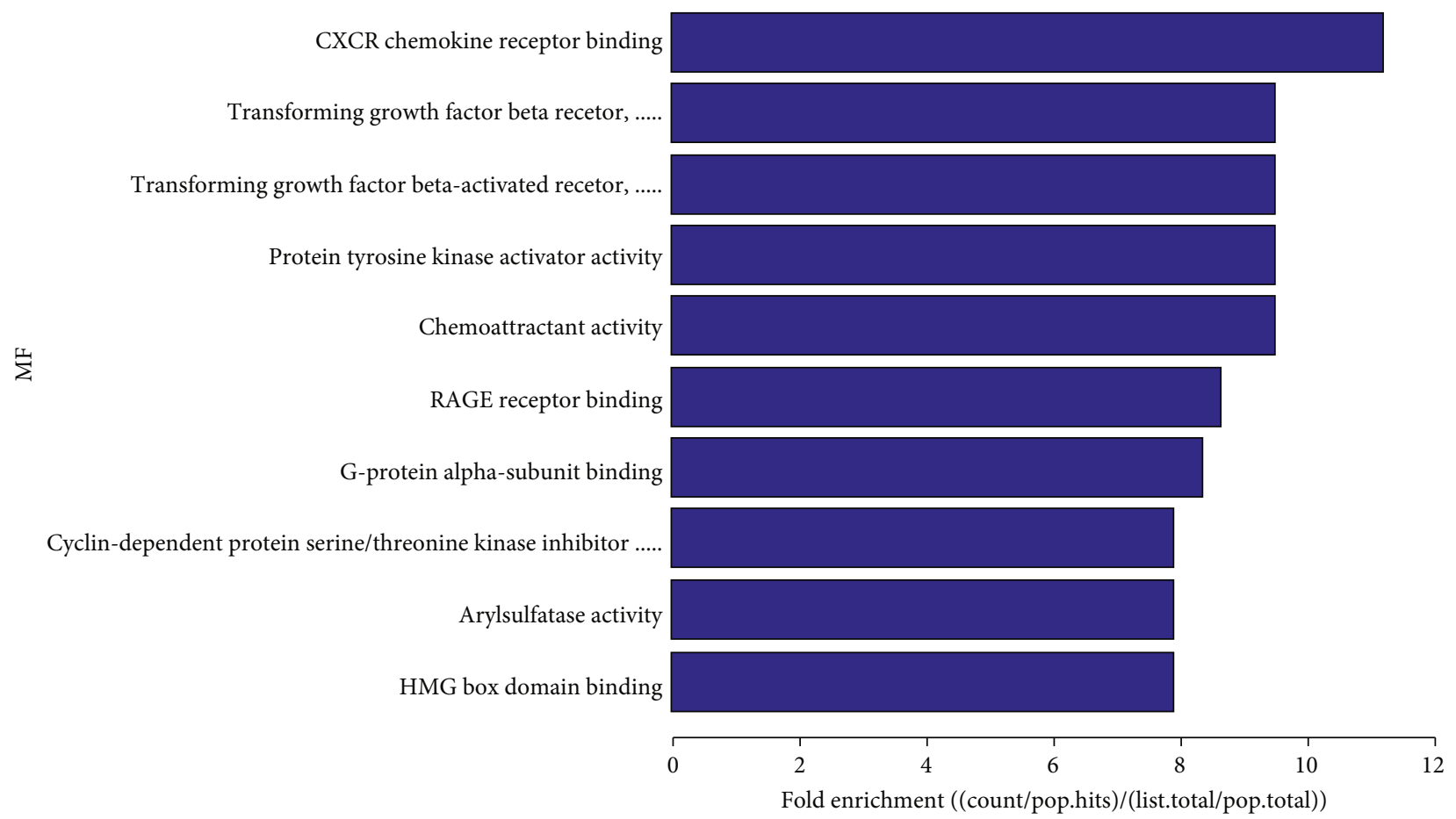

(c)

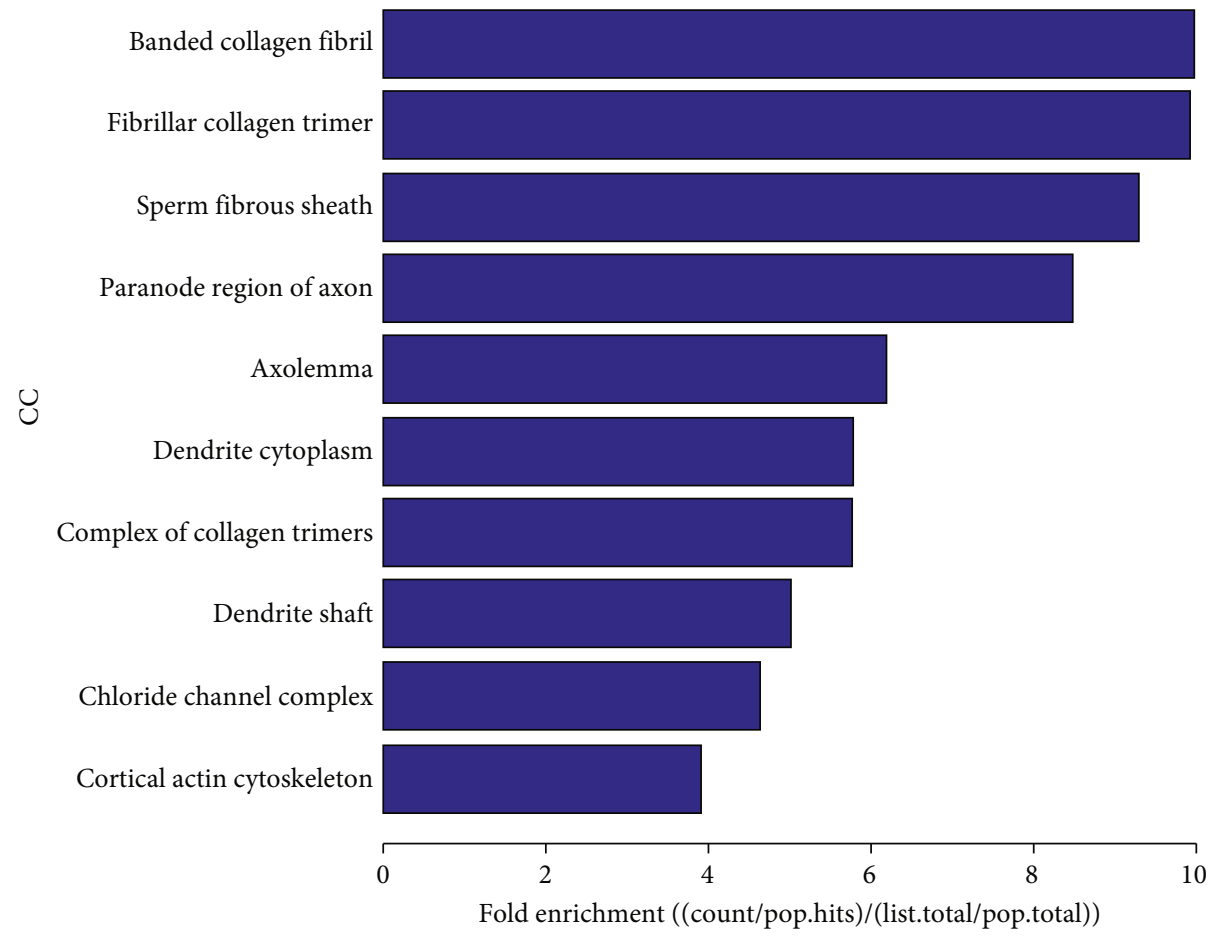

(d)

Figure 4: Continued. 


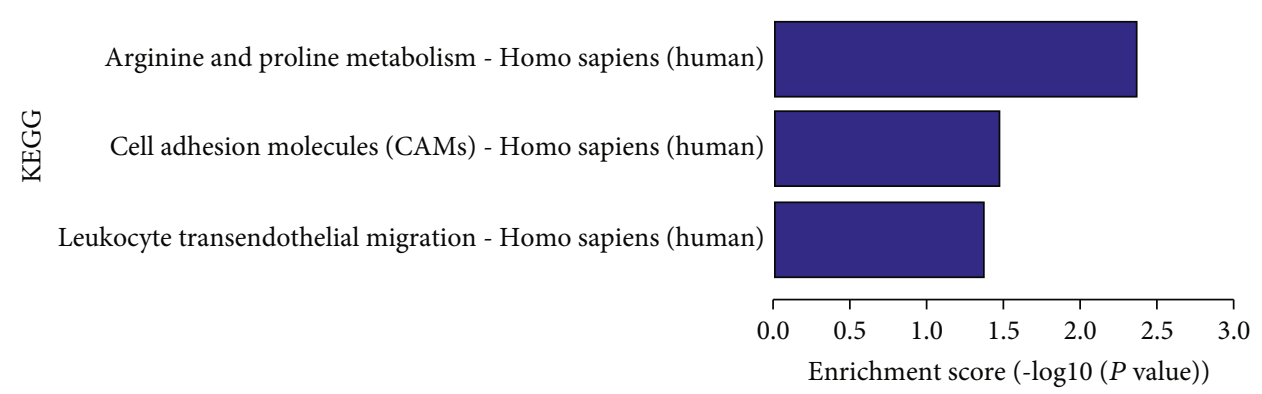

(e)

FIGURE 4: Functional analysis of the top 10 DElncRNAs associated with DEmRNAs between strained HPDLSCs and PPDLSCs. (a) lncRNAmRNA interaction network of the top 10 DElncRNAs related to mRNAs. The red and blue spots represent upregulated lncRNAs and downregulated lncRNAs, respectively. In addition, the green and pink colors represent upregulated coding genes and downregulated coding genes, respectively. Top 10 biological processes (b), molecular functions (c), and cellular components (d) of the differences in coexpressed lncRNAs. (e) KEGG pathways of the differences in coexpressed lncRNAs.

Excessive mechanical stimuli can cause irreversible damage to PDLSCs, especially to those in an inflammatory state [23]. However, studies on the expression of lncRNAs involved in strain-induced PDLSCs and their potential effects on cellular functions are limited. In this study, we identified thousands of DElncRNAs and DEmRNAs in HPDLSCs and PPDLSCs after SMS application, and various lncRNAs and mRNAs were found to be solely expressed in strained HPDLSCs or PPDLSCs, indicating that different mechanisms may be involved in the mechanotransductive responses of PDLSCs derived from different contexts.

Using microarray analysis, we observed that DElncRNAs in strained HPDLSCs were mainly enriched in mechanoconductive processes; pathological pathways such as cell-type apoptotic process and regulation of neuron apoptotic process were associated with dysregulated lncRNAs in strained PPDLSCs. In addition, based on KEGG pathway analysis, the DElncRNAs are largely related to pathological conditions such as Huntington's disease [24], bladder cancer [25], and non-small-cell lung cancer [26]. Therefore, cell functions regulated by lncRNAs have a potential role in PDLSCs, and aberrant lncRNA transcripts are associated with periodontitis progression [27].

Cytoskeletal dynamics and integrity are of vital importance for cell differentiation commitment, by which the bone loss occurring in periodontitis can be alleviated [28]. By altering the expression of eukaryotic cytoskeleton proteins, which play important roles in cancer progression and cytoskeleton modulation, KIF20A is sensitive to alterations along with mechanical loadings [29]. In this study, KIF20A was most downregulated in strained PPDLSCs, suggesting that dysregulated mRNAs possibly modulate expression through interactions with intracellular cytoskeleton mechanisms. Therefore, further functional validation of these dysregulated transcripts in periodontitis is warranted.

Furthermore, to identify potential lncRNAs associated with strained PDLSCs, we integrated the coexpression networks of lncRNAs and mRNAs. A total of 1,250 pairs based on the top 10 dysregulated lncRNAs between strained PPDLSCs and HPDLSCs were established. Of those, RP11597D13.9, an antisense lncRNA, correlated with up to 160
mRNAs, and it may affect a nearby coding gene: FAM198B [30]. FAM198B has been implicated as a tumor inhibitor, attenuating lung cancer cell invasion and thus improving the overall survival of patients with lung adenocarcinoma [31]. In our study, RP11-597D13.9 displayed an inverse trend of downregulation in strained PPDLSCs, indicating a possible pathological state for these cells. Moreover, lncRNA XIST has long been recognized as an oncogenic gene and is preferentially expressed in cancers $[32,33]$. LPS-induced inflammation can increase the levels of XIST expression, which in turn suppresses acute inflammation via MAPK signaling [34]. Contrary to these results, there was a significant decrease in XIST in strained PPDLSCs together with CH1CI (Brx), one of the major downstream target genes for XIST [35]. In our study, we first found that lncRNA-XIST expression decreased in PDLSCs derived from an inflammatory microenvironment. Additionally, although SMS elongation significantly decreased the expression of lncRNA-XIST in PPDLSCs compared with HPDLSCs, the upregulation of lncRNA-XIST in strained PPDLSCs increased the process of osteogenic differentiation, indicating that lncRNA-XIST may be one of the nonnegligible reasons for the impaired osteogenesis in strained PPDLSCs.

\section{Conclusions}

In summary, differentially expressed lncRNA profiles between HPDLSCs and PDLSCs under mechanical exposure were first identified in this study, and many were specifically expressed. By functional analysis, we confirmed that DE transcripts in PPDLSCs participate in many pathological processes and might be involved in regulating periodontitis progression under tension. In our study, we found that the expression of lncRNA-XIST obviously decreased in PPDLSCs, and the osteogenic ability of PPDLSCs under tension loading was significantly upregulated after upregulation of lncRNA-XIST by lentivirus. These results hint us that lncRNAs could regulate the osteogenic ability of PDLSCs under tension loading. Although some lncRNAs are predicted, comprehensive analyses are still needed to elucidate the details of the relevant molecular mechanisms. 


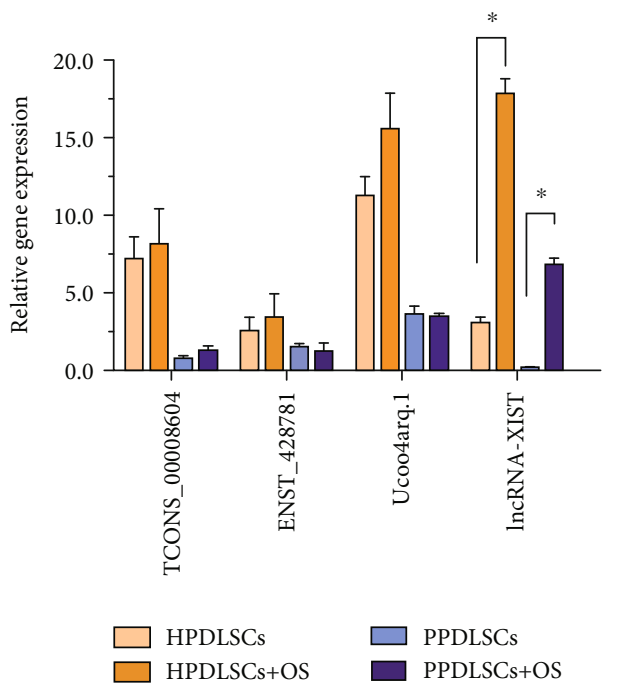

(a)

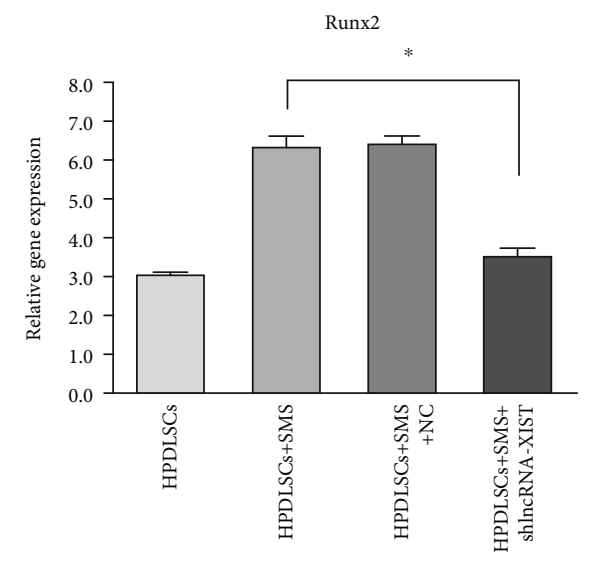

(c)

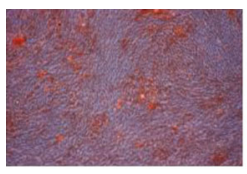

HPDLSCs

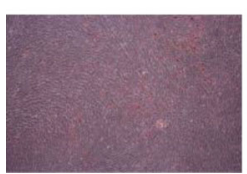

PPDLSCs

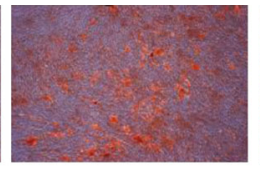

HPDLSCs+SMS

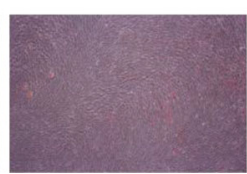

PPDLSCs+SMS

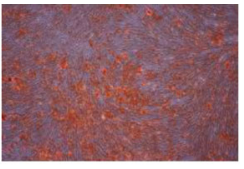

HPDLSCs+SMS+NC

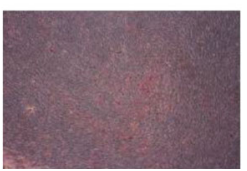

PPDLSCs+SMS+NC

(e)

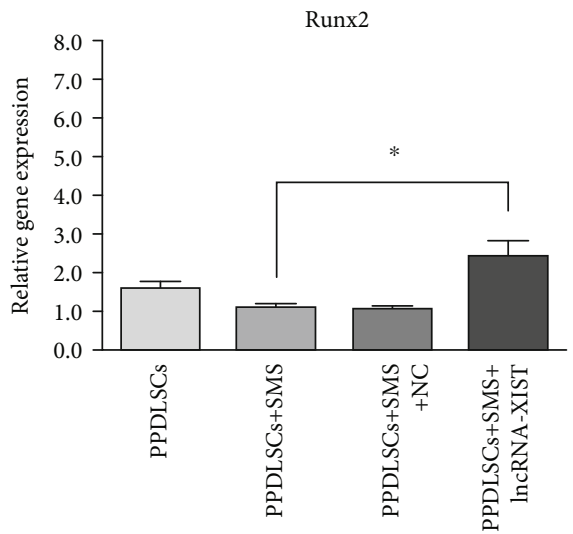

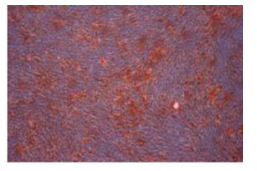

HPDLSCs+SMS+ shlncRNA-XIST

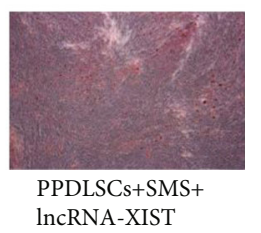

(d)

(b)

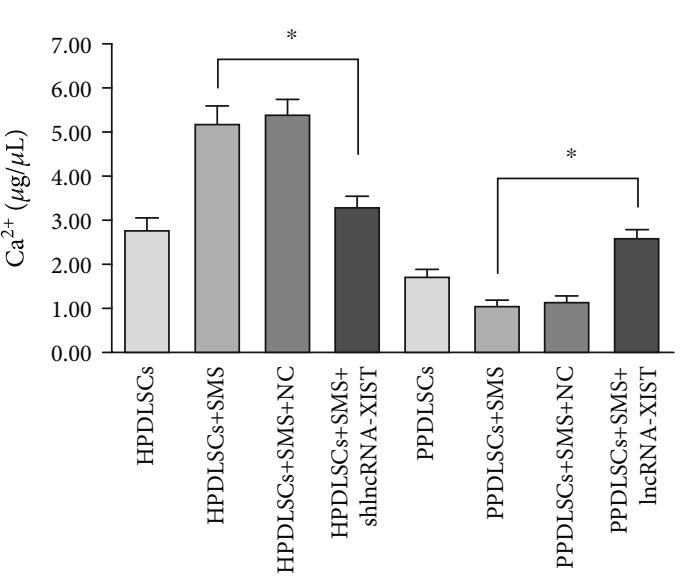

(f)

FIgURE 5: The expression levels of DElncRNAs in HPDLSCs and PPDLSCs during osteogenic differentiation: (a) the expression levels of DElncRNAs in HPDLSCs and PPDLSCs after osteogenic differentiation for 7 days; (b) the expression levels of lncRNA-XIST in strained HPDLSCs and PPDLSCs; (c) detection of the osteogenic gene Runx2 in strained HPDLSCs infected with shlncRNA-XIST; (d) detection of the osteogenic gene Runx2 in strained PPDLSCs infected with lncRNA-XIST; (e) alizarin red staining for HPDLSCs and PPDLSCs; (f) calcium quantification for HPDLSCs and PPDLSCs. All experiments were performed in triplicate, and the data are presented as the mean \pm S.D. Scale bar $=100 \mu \mathrm{m}$. 


\section{Data Availability}

The data used to support the findings of this study are available from the corresponding author upon request.

\section{Conflicts of Interest}

The authors declare no potential competing interests.

\section{Authors' Contributions}

Jia Liu, Yan Zhao, and Qiannan Niu contributed equally to this work.

\section{Acknowledgments}

This work was supported by the National Natural Science Foundation of China (grant no. 81701002 and 81970960) and the International Scientific and Technological Cooperation and Exchange Program in Shaanxi Province of China (No. 2021KW45).

\section{References}

[1] J. B. Guo, J. Q. Weng, Q. Rong et al., "Investigation of multipotent postnatal stem cells from human maxillary sinus membrane," Scientific Reports, vol. 5, no. 1, article 11660, 2015.

[2] T. Crotti, M. D. Smith, R. Hirsch et al., "Receptor activator NF kappaB ligand (RANKL) and osteoprotegerin (OPG) protein expression in periodontitis," Jouranl of Periodontal Research, vol. 38, no. 4, pp. 380-387, 2003.

[3] C. Ren, C. McGrath, M. Gu et al., "Low-level laser-aided orthodontic treatment of periodontally compromised patients: a randomised controlled trial," Lasers in Medical Science, vol. 35, no. 3, pp. 729-739, 2020.

[4] R. X. Wu, C. S. Bi, Y. Yu, L. L. Zhang, and F. M. Chen, "Age-related decline in the matrix contents and functional properties of human periodontal ligament stem cell sheets," Acta Biomaterialia, vol. 22, pp. 70-82, 2015.

[5] J. Liu, L. Y. Wang, W. J. Liu, Q. Li, Z. Jin, and Y. Jin, “Dental follicle cells rescue the regenerative capacity of periodontal ligament stem cells in an inflammatory microenvironment," PLoS One, vol. 9, no. 10, article e108752, 2014.

[6] J. Liu, Q. Li, S. Y. Liu et al., "Periodontal ligament stem cells in the periodontitis microenvironment are sensitive to static mechanical strain," Stem Cells International, vol. 2017, Article ID 1380851, 13 pages, 2017.

[7] M. Wolf, S. Lossdörfer, P. Römer et al., "Short-term heat pretreatment modulates the release of HMGB1 and proinflammatory cytokines in hPDL cells following mechanical loading and affects monocyte behavior," Clinical Oral Investigations, vol. 20, no. 5, pp. 923-931, 2016.

[8] R. Jia, Y. J. Yi, J. Liu et al., "Cyclic compression emerged dual effects on the osteogenic and osteoclastic status of LPSinduced inflammatory human periodontal ligament cells according to loading force," BMC Oral Health, vol. 20, no. 1, p. 7, 2020.

[9] J. S. Mattick, "RNA regulation: a new genetics?," Nature Reviews Genetics, vol. 5, no. 4, pp. 316-323, 2004.
[10] L. W. Harries, "Long non-coding RNAs and human disease," Biochemical Society Transactions, vol. 40, no. 4, pp. 902-906, 2012.

[11] Y. G. Zou, C. Li, F. P. Shu et al., "IncRNA expression signatures in periodontitis revealed by microarray: the potential role of IncRNAs in periodontitis pathogenesis," Journal of Cellular Biochemistry, vol. 116, no. 4, pp. 640-647, 2015.

[12] W. Peng, W. Deng, J. Zhang, G. W. Pei, Q. Rong, and S. X. Zhu, "Long noncoding RNA ANCR suppresses bone formation of periodontal ligament stem cells via sponging miRNA758," Biochemical and Biophysical Research Communications, vol. 503, no. 2, pp. 815-821, 2018.

[13] L. Wang, F. Wu, Y. Song et al., "Long noncoding RNA related to periodontitis interacts with miR-182 to upregulate osteogenic differentiation in periodontal mesenchymal stem cells of periodontitis patients," Cell Death \& Disease, vol. 7, no. 8, article e2327, 2016.

[14] R. R. Meng, M. Song, and J. S. Pan, "Rho is involved in periodontal tissue remodelling with experimental tooth movement in rats," Archives of Oral Biology, vol. 60, no. 6, pp. 923-931, 2015.

[15] W. Liu, Y. B. Cao, L. Dong et al., "Periodontal therapy for primary or secondary prevention of cardiovascular disease in people with periodontitis," The Cochrane Database of Systematic Reviews, vol. 12, no. 12, article CD009197, 2019.

[16] Z. H. Yang, F. Jin, X. J. Zhang et al., "Tissue engineering of cementum/periodontal-ligament complex using a novel three-dimensional pellet cultivation system for human periodontal ligament stem cells," Tissue Engineering. Part C. Methods, vol. 15, no. 4, pp. 571-581, 2009.

[17] T. D. Schmittgen and K. J. Livak, "Analyzing real-time PCR data by the comparative C(T) method," Nature Protocals, vol. 3, no. 6, pp. 1101-1108, 2008.

[18] M. Guttman and J. L. Rinn, "Modular regulatory principles of large non-coding RNAs," Nature, vol. 482, no. 7385, pp. 339346, 2012.

[19] R. Chen, J. Liu, M. Xiao, F. Wang, and X. Lin, "Microarray expression profile analysis of long noncoding RNAs in premature brain injury: a novel point of view," Neuroscience, vol. 319, pp. 123-133, 2016.

[20] M. A. Pujana, J. D. Han, L. M. Starita et al., "Network modeling links breast cancer susceptibility and centrosome dysfunction," Nature Genetics, vol. 39, no. 11, pp. 13381349, 2007.

[21] X. Q. Zhang, L. H. Ren, X. Y. Yan et al., "Identification of immune-related lncRNAs in periodontitis reveals regulation network of gene-lncRNA-pathway-immunocyte," International Immunopharmacology, vol. 84, article 106600, 2020.

[22] Y. Liu, C. P. Liu, A. K. Zhang et al., "Down-regulation of long non-coding RNA MEG3 suppresses osteogenic differentiation of periodontal ligament stem cells (PDLSCs) through miR27a-3p/IGF1 axis in periodontitis," Aging, vol. 11, no. 15, pp. 5334-5350, 2019.

[23] C. X. Zhang, Y. Q. Lu, L. K. Zhang et al., "Influence of different intensities of vibration on proliferation and differentiation of human periodontal ligament stem cells," Archives of Medical Science. AMS, vol. 3, no. 3, pp. 638-646, 2015.

[24] R. Johnson, "Long non-coding RNAs in Huntington's disease neurodegeneration," Neurobiology of Disease, vol. 46, no. 2, pp. 245-254, 2012. 
[25] Y. H. Zhan, Z. C. Chen, S. M. He et al., "Long non-coding RNA SOX2OT promotes the stemness phenotype of bladder cancer cells by modulating SOX2," Molecular Cancer, vol. 19, no. 1, p. 25, 2020.

[26] F. Li, Q. Y. Zhang, Y. G. Gong, and J. X. Yu, "The lncKLF6/KLF6 feedback loop regulates the growth of nonsmall cell lung cancer," American Journal of Cancer Research, vol. 8, no. 8, pp. 1427-1439, 2018.

[27] Y. D. Liu, Q. F. Liu, Z. P. Li et al., "Long non-coding RNA and mRNA expression profiles in peri-implantitis vs periodontitis," Journal of Periodontal Research, vol. 55, no. 3, pp. 342$353,2020$.

[28] I. Binderman, N. Gadban, and A. Yaffe, "Cytoskeletal disease: a role in the etiology of adult periodontitis," Oral Diseases, vol. 20, no. 1, pp. 10-16, 2014.

[29] J. Schiewek, U. Schumacher, T. Lange et al., "Clinical relevance of cytoskeleton associated proteins for ovarian cancer," Journal of Cancer Research and Clinical Oncology, vol. 144, no. 11, pp. 2195-2205, 2018.

[30] H. Wang, Z. Y. Fu, C. C. Dai et al., "LncRNAs expression profiling in normal ovary, benign ovarian cyst and malignant epithelial ovarian cancer," Scientific Reports, vol. 6, no. 1, article 38983, 2016.

[31] C. Y. Hsu, G. C. Chang, Y. J. Chen et al., "FAM198B is associated with prolonged survival and inhibits metastasis in lung adenocarcinoma via blockage of ERK-mediated MMP-1 expression," Clinical Cancer Research, vol. 24, no. 4, pp. 916926, 2018.

[32] N. N. Sun, G. Z. Zhang, and Y. Y. Liu, "Long non-coding RNA XIST sponges miR-34a to promotes colon cancer progression via Wnt/ $\beta$-catenin signaling pathway," Gene, vol. 665, pp. 141-148, 2018.

[33] Y. Zhang, H. Zhang, W. Zhang, Y. J. Zhang, W. Wang, and L. Nie, "LncRNA XIST modulates 5-hydroxytrytophaninduced visceral hypersensitivity by epigenetic silencing of the SERT gene in mice with diarrhea-predominant IBS," Cellular Signalling, vol. 73, article 109674, 2020.

[34] B. B. Shenoda, S. Ramanathan, R. Gupta et al., "Xist attenuates acute inflammatory response by female cells," Cellular and Molecular Life Sciences, vol. 78, no. 1, pp. 299-316, 2021.

[35] M. C. Simmler, E. Heard, C. Rougeulle, C. Cruaud, J. Weissenbach, and P. Avner, "Localization and expression analysis of a novel conserved brain expressed transcript, Brx/BRX, lying within the Xic/XIC candidate region," Mammalian Genome, vol. 8, no. 10, pp. 760-766, 1997. 